Marquette University

e-Publications@Marquette

College of Nursing Faculty Research and

Publications

Nursing, College of

$5-2016$

\title{
Probiotics for Treatment and Prevention of Urogenital Infections in Women: A Systematic Review
}

\author{
Lisa Hanson \\ Marquette University, lisa.hanson@marquette.edu \\ Leona VandeVusse \\ Marquette University, leona.vandevusse@marquette.edu \\ Martha G. Jermé \\ Marquette University, martha.jerme@marquette.edu \\ Cybéle L. Abad \\ Nasia Safdar \\ University of Wisconsin - Madison
}

Follow this and additional works at: https://epublications.marquette.edu/nursing_fac

Part of the Nursing Commons

\section{Recommended Citation}

Hanson, Lisa; VandeVusse, Leona; Jermé, Martha G.; Abad, Cybéle L.; and Safdar, Nasia, "Probiotics for Treatment and Prevention of Urogenital Infections in Women: A Systematic Review" (2016). College of Nursing Faculty Research and Publications. 459.

https://epublications.marquette.edu/nursing_fac/459 
Marquette University

\section{e-Publications@Marquette}

\section{Nursing Faculty Research and Publications/College of Nursing}

This paper is NOT THE PUBLISHED VERSION; but the author's final, peer-reviewed manuscript. The published version may be accessed by following the link in the citation below.

Journal of Midwifery \& Women's Health, Vol. 61, No. 3 (May/June 2016): 339-355. DOI. This article is (C) Wiley and permission has been granted for this version to appear in e-Publications@Marquette. Wiley does not grant permission for this article to be further copied/distributed or hosted elsewhere without the express permission from Wiley.

\section{Probiotics for Treatment and Prevention of Urogenital Infections in Women: A Systematic Review}

\section{Lisa Hanson}

College of Nursing, Marquette University, Milwaukee, WI

\section{Leona VandeVusse}

College of Nursing, Marquette University, Milwaukee, WI

\section{Martha Jerme}

Raynor Memorial Libraries, Marquette University, Milwaukee, WI

\section{Cybele L. Abad}

University if the Philippines, Philippine General Hospital, Manila

\section{Nasia Safdar}

Wisconsin School of Medicine and Public Health, Department of Medicine and Infectious Disease, Madison, WI 


\section{Abstract}

\section{Introduction}

Probiotics are a complementary and integrative therapy useful in the treatment and prevention of urogenital infections in women. This study extends the work of researchers who systematically investigated the scientific literature on probiotics to prevent or treat urogenital infections.

\section{Methods}

A systematic review was conducted to determine the efficacy of probiotics for prevention and/or treatment of urogenital infections in adult women from January 1, 2008, through June 30, 2015. We searched in CINAHL, MEDLINE, Cochrane Central Register of Controlled Trials, Web of Science, Dissertations and Theses, and Alt-HealthWatch. After removing duplicates and studies that did not meet inclusion criteria, 20 studies were reviewed. All included at least one species of Lactobacillus probiotic as an intervention for treatment or prevention of urogenital infections. Data extracted included samples, settings, study designs, intervention types, reported outcomes, follow-up periods, and results. We evaluated all randomized controlled trials for risk of bias and made quality appraisals on all studies.

Results

Fourteen of the studies focused on bacterial vaginosis (BV), 3 on urinary tract infections (UTIs), 2 on vulvovaginal candidiasis, and one on human papillomavirus (HPV) as identified on Papanicolaou test. Studies were heterogeneous in terms of design, intervention, and outcomes. Four studies were of good quality, 9 of fair, and 7 poor. Probiotic interventions were effective for treatment and prevention of BV, prevention of recurrences of candidiasis and UTIs, and clearing HPV lesions. No study reported significant adverse events related to the probiotic intervention.

\section{Discussion}

The quality of the studies in this systematic review varied. Although clinical practice recommendations were limited by the strength of evidence, probiotic interventions were effective in treatment and prevention of urogenital infections as alternatives or co-treatments. More good quality research is needed to strengthen the body of evidence needed for application by clinicians.

\section{INTRODUCTION}

Urogenital infections present significant problems for women's health care providers. Although effective antimicrobial treatments are readily available in developed countries, concerns mount about antibiotic cost, overuse or overexposure, sensitivity, and sequelae, including antibiotic resistance. $\frac{1}{}$ An integrated approach to preventing recurrences of urogenital infections is desirable to enhance the health and well-being of women and to decrease the need for repeat doses of antibiotics or other major interventions.

According to the Centers for Disease Control and Prevention (CDC), $\underline{2}$ bacterial vaginosis (BV) is the most common form of vaginitis in premenopausal women. The vagina is normally a microbial environment dominated by Lactobacillus bacteria. $\underline{3}$ BV is the result of a shift to a mixture of primarily anaerobic bacterial species. $\underline{3}$ The species associated with BV include Gardnerella vaginalis, Ureaplasma, and Mycoplasma, among others. $\stackrel{?}{-}$ There are several antibiotics recommended and alternative BV treatment 
options available with oral or vaginal route options. $\stackrel{2}{ }$ However, recurrences of BV are common and multifactorial. $\underline{4}$

Vulvovaginal candidiasis results from an overgrowth of one or more types of yeast organisms that inhabit the vaginal mucosa in small numbers, most commonly Candida albicans.. Symptoms include external dysuria, pruritus, redness, and floccular vaginal discharge. $\frac{5}{2}$ Recurrences are common, especially in women with risk factors, such as diabetes mellitus or immune system compromise. However, it is estimated that $75 \%$ of women will experience at least one episode of vulvovaginal candidiasis at some point in their lives. $\underline{5}$

Escherichia coli is the organism responsible for the largest proportion of symptomatic and asymptomatic urinary tract infections (UTIS) in women. $\underline{6}$ Ideally, antibiotic treatment is reserved for symptomatic patients (or asymptomatic pregnant women) in whom the organism grows as a single isolate of greater than $100,000\left(10^{5}\right)$ colony-forming units (CFUs) on a clean catch midstream urine culture. ${ }^{6}$ In clinical practice, confirmatory urine culture is often sent while treatment commences. Use of antibiotics with low patterns of resistance is recommended for first-line management. $\underline{6}$

Human papillomavirus (HPV) is the cause of nearly all abnormal Papanicolaou tests. Low-grade squamous intraepithelial lesions (LGSILs) are associated with more high-risk forms of HPV. Expression of HPV is transient. Viral clearance can lead to resolution over a period of several months, resulting in normal cervical cytology on reexamination. ${ }^{?}$

\section{Quick Points}

- Probiotic lactobacilli are commonly used choices for treating and preventing urogynecologic infections.

- Although clinical practice recommendations were limited by the strength of evidence, probiotic interventions appear to be effective in treatment and prevention of urogenital infections as an alternative or co-treatment.

- None of the probiotic interventions were associated with serious adverse events.

- More well-designed clinical research studies are needed on probiotics used to treat or prevent urogenital infections in women.

- If used as co-treatments, other evidence suggests that antibiotic and probiotic interventions should be separated by at least 2 to 4 hours to avoid the destruction of the live microorganisms in the gastrointestinal tract.

There is a clear link between vaginal microflora and urogenital infections. Independent of personal hygiene, the large varieties of microbes that populate the vagina originate from the gastrointestinal tract. 8 Healthy vaginal microflora is characterized by a dominance of Lactobacillus bacterial species. $\frac{9,10}{}$ A number of scientific investigations have demonstrated that the gastrointestinal and vaginal microflora can be modified with probiotic supplementation to treat and prevent genitourinary infections. $\frac{10}{10}$ Probiotics are live bacteria that confer a health effect on the host when they are administered in sufficient amounts. $\frac{11}{}$ Therefore, probiotics used to prevent and treat genitourinary infections contain Lactobacillus species, since the target is the vaginal microflora. The mechanisms of 
action of probiotics include acidification of the mucosal surface, prevention of the adherence of pathogens, the production of substances such as vitamins and immune modulators, and synergistic action with the host immune system. $\underline{10}$ Some species of Lactobacillus produce hydrogen peroxide, which further acidifies the vaginal mucosa. $\underline{10}$ This property makes Lactobacillus a common probiotic choice for treating and preventing urogynecologic infections.

Abad and Safdar $\underline{12}$ conducted a systematic review of the literature from inception through December 2007 to identify the role of Lactobacillus-containing probiotics in the prevention or treatment of 3 specific urogenital infections: vulvovaginal candidiasis, UTI, and BV. They found evidence for the benefits of certain Lactobacillus strains (particularly $L$ rhamnosus GR-1 and $L$ reuteri) for prevention and treatment of recurrent urogenital infection, particularly for BV. They also found limited data on the use of probiotics for UTI and candidiasis. Since their systematic review, there has been significant consumer and scientific interest in the use of probiotics. We extended that systematic review with contemporary scientific literature on probiotics to treat or prevent urogenital infections in adult women.

\section{METHODS}

We conducted literature searches in CINAHL, MEDLINE, Cochrane Central Register of Controlled Trials, Web of Science, Dissertations and Theses, and Alt-HealthWatch. Search terms included "probiotics," "Lactobacillus," "Bifidobacterium," and "Saccharomyces" combined with

"urogenital infections," "vaginitis," "vaginosis," "vulvovaginitis," vulvovaginal candidiasis," "urinary tract infections," "vaginal discharge," "reproductive tract infections," "sexually transmitted diseases," "papillomavirus infections," and "urethritis." Results were limited to quantitative studies on adult females that tested probiotics to prevent or treat common urogynecologic infections and were published in English from January 2008 through June 2015. Articles addressing HIV infections, cancer, major diagnoses such as diabetes mellitus, and women who were pregnant were excluded from the studies reviewed. The first 3 authors also reviewed reference lists of included articles and subsequent published reviews that addressed some but not all of our inclusion criteria for relevant papers to identify additional studies not retrieved through the initial search.

The Preferred Reporting Items for Systematic Reviews and Meta-analyses (PRISMA) $\underline{13}$ guidelines were followed throughout the systematic review. The PRISMA Diagram (Figure $\underline{1}$ ) outlines the process used to identify, screen, and determine the eligibility of the articles to be analyzed. Of the 40 full-text articles screened, 20 were excluded with reasons. Twenty studies were included in the final analysis. These included 14 randomized controlled trials (RCTs), $\underline{3,14-26}$ one quasi-experiment, $\underline{27} 2$ prospective cohort studies, $\underline{28,29}$ and 3 single-group investigations. $\underline{\underline{30-32}}$ 


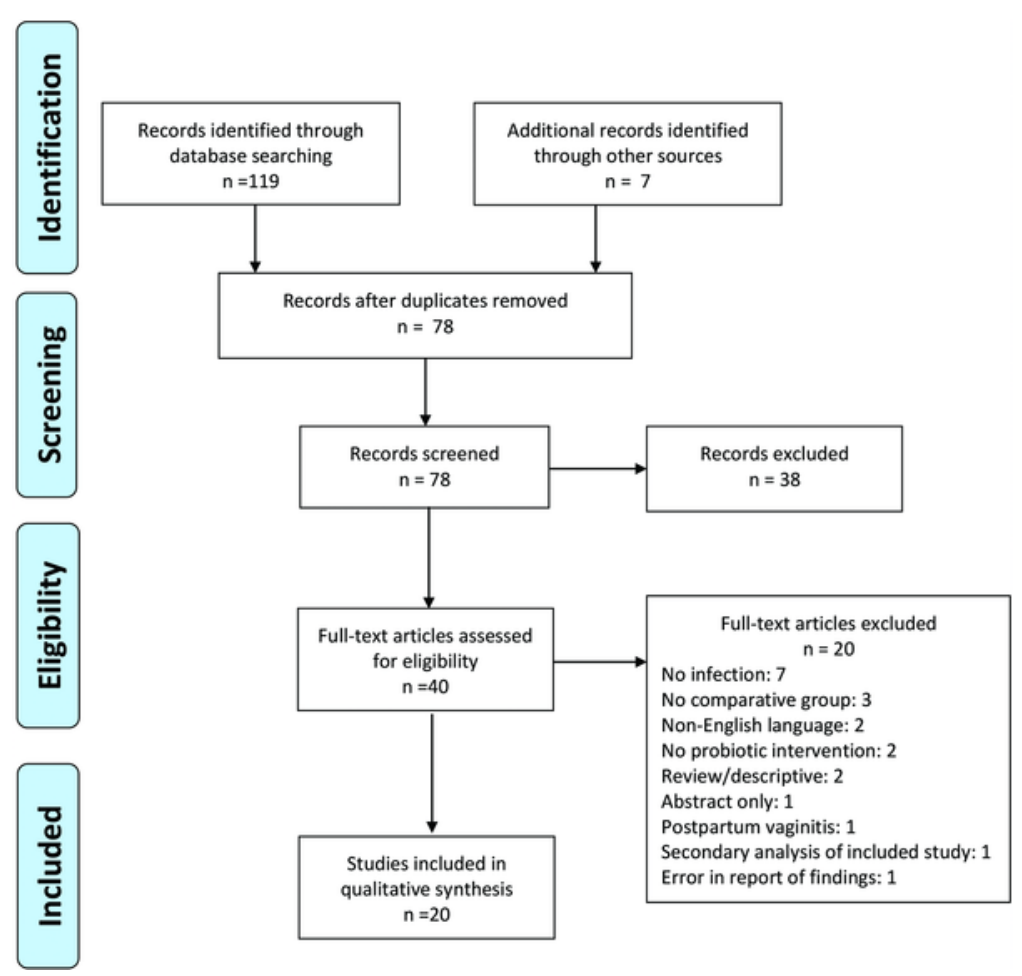

Figure 1. PRISMA Flow Diagram

Data were extracted independently and reviewed by the first 2 authors. Data collected included samples, settings, study designs, intervention types, reported outcomes, follow-up periods, and results. Data extraction was verified by comparing data tables. Statistical analysis was not possible because of the heterogeneity of probiotic preparations, primary and secondary outcomes, and clinical trial methods. Instead, a detailed quality assessment was made on each study. We adapted an assessment approach that allowed for a broader evaluation of quality. $\frac{33}{3}$ First, the risk of bias was assessed for the 14 RCTs using the Cochrane Collaboration Risk of Bias of Randomized Controlled Trials Assessment, $\underline{34}$ which includes details about randomization and blinding. The first 2 authors conducted these evaluations and rated each study as low, unclear, or high risk of bias. $\frac{34}{\text { Agreement was reached }}$ about any differing ratings. These risk of bias ratings are presented in Table $\underline{1}$. These ratings were shared with the last 2 authors, who individually evaluated every study on the following additional 3 domains recommended by the Agency for Healthcare Research and Quality (AHRQ) for evaluating effectiveness: consistency of the findings, directness, and precision. $\underline{35}$ The non-RCTs were rated on only these final 3 domains, while the RCTs were rated on all of the criteria. The last 2 authors reached consensus on their combined evaluations of each study using both of the quality assessment approaches noted above. Finally, they rated the study's overall quality as a) "good" (low risk of bias for RCTs, consistency of results), direct (findings directly attributable to intervention or not), and precise (repeatability); b) "fair" (variable on these domains); or c) "poor" (high risk of bias for RCTs, inconsistent, findings indirectly related to the intervention, and imprecise), $\frac{33-35}{2}$ as also shown in Table 1. 
Table 1. Summary of Studies of Probiotics by Urogenital Infections

\begin{tabular}{|c|c|c|c|c|c|}
\hline $\begin{array}{l}\text { Author } \\
\text { Year } \\
\text { Country }\end{array}$ & $\begin{array}{l}\text { Design } \\
\text { Total N }\end{array}$ & $\begin{array}{l}\text { Risk of Bias } \\
\text { Rating } \\
\text { Overall } \\
\text { Quality } \\
\text { Rating }\end{array}$ & $\begin{array}{l}\text { Intervention } \\
\text { Probiotic Species, } \\
\text { Dose, Route, Duration, } \\
\text { and Follow-up }\end{array}$ & $\begin{array}{l}\text { Medication } \\
\text { Pretreatment } \\
\text { or Cotreatment }\end{array}$ & $\begin{array}{l}\text { Probiotic Intervention Findings } \\
\text { ( } P \text { values provided if available) }\end{array}$ \\
\hline \multicolumn{6}{|c|}{$\begin{array}{l}\text { Bacterial Vaginosis } \\
\text { and Vulvovaginal } \\
\text { Candidiasis }\end{array}$} \\
\hline $\begin{array}{l}\text { Ehrstrom }{ }^{3} \\
2010 \\
\text { Sweden }\end{array}$ & $\begin{array}{l}\text { RCT } \\
96\end{array}$ & $\begin{array}{l}\text { Unclear } \\
\text { Poor }\end{array}$ & $\begin{array}{l}\text { L gasseri LN40 } \\
\text { L fermentum LN99 } \\
\text { L casei subsp. rhamnosus LN113 } \\
\text { Pediococcus acidilactici LN23 } \\
\text { 108-109 total; vaginal capsules; } \\
5 \text { days; } 6 \text { months }\end{array}$ & $\begin{array}{l}\text { Metronidazole or } \\
\text { clotrimazole }\end{array}$ & $\begin{array}{l}\uparrow \text { colonization with lactic acid } \\
\text { bacteria }(P<.0001) \\
\downarrow \text { symptoms after administration }(P \\
=.03) \text { and after second menses }(P= \\
.04)\end{array}$ \\
\hline \multicolumn{6}{|l|}{ Bacterial Vaginosis } \\
\hline $\begin{array}{l}\text { Hemmerling }{ }^{14} \\
2010 \\
\text { US }\end{array}$ & $\begin{array}{l}\text { RCT } \\
24\end{array}$ & $\begin{array}{l}\text { Low } \\
\text { Good }\end{array}$ & $\begin{array}{l}\text { Product }{ }^{\text {a }} \text { containing } L \text { crispatus } \\
(\text { CTV-05) } \\
2 \times 109 ; \text { vaginal applicator; } 2 \\
\text { weeks; } 1 \text { month }\end{array}$ & Metronidazole & $\begin{array}{l}61 \% \text { of probiotic group colonized } \\
\text { with Lactin- } V \text { by day } 10 \text { or } 28 \\
\text { No significant difference in } A E\end{array}$ \\
\hline $\begin{array}{l}\text { Mastromarino }{ }^{15} \\
2009 \\
\text { Italy }\end{array}$ & $\begin{array}{l}\mathrm{RCT} \\
34\end{array}$ & $\begin{array}{l}\text { Unclear } \\
\text { Fair }\end{array}$ & $\begin{array}{l}\text { Combination product }^{\mathrm{b}} \\
\text { containing } \\
L \text { brevis } \mathrm{CD} 2 \\
L \text { salivarius subsp salicinius } \\
L \text { plantarum } \\
\text { Ascorbic acid } \\
\text { Sodium bicarbonate } \\
\text { Acetic acid } \\
\text { Stearic acid } \\
\text { Magnesium stearate } \\
\leq 109 ; \text { vaginal tablets; } 7 \text { days; } 3 \\
\text { weeks }\end{array}$ & None & $\begin{array}{l}\uparrow \text { BV cure rate at } 2 \text { weeks }(P<.05) \\
\downarrow \text { Nugent scores }(P<.05)\end{array}$ \\
\hline $\begin{array}{l}\text { Hemalatha } \underline{16} \\
2012 \\
\text { India }\end{array}$ & $\begin{array}{l}\text { RCT } \\
149\end{array}$ & $\begin{array}{l}\text { Low } \\
\text { Fair }\end{array}$ & $\begin{array}{l}\text { Combination product }^{\mathrm{b}} \\
\text { containing } \\
L \text { brevis CD2 }\end{array}$ & none & $\begin{array}{l}\downarrow \text { proinflammatory cytokines IL- } \beta \\
(P<.001), \text { IL- } 6(P<.015)\end{array}$ \\
\hline
\end{tabular}




\begin{tabular}{|c|c|c|c|c|c|}
\hline & & & $\begin{array}{l}\text { L salivarius subsp. salicinius } \\
\text { L plantarum } \\
\text { Ascorbic acid } \\
\text { Sodium bicarbonate } \\
\text { Acetic acid } \\
\text { Stearic acid } \\
\text { Magnesium stearate } \\
\leq 109 ; \text { vaginal tablets; } 8 \text { days; } 9 \\
\text { days }\end{array}$ & & \\
\hline $\begin{array}{l}\text { Petricevic }{ }^{17} \\
2008 \\
\text { Austria }\end{array}$ & $\begin{array}{l}\text { RCT } \\
190\end{array}$ & $\begin{array}{l}\text { Unclear } \\
\text { Fair }\end{array}$ & $\begin{array}{l}\text { Combination product }{ }^{c} \\
\text { containing } \\
\text { L casei rhamnosus (Lcr35) } \\
3.41 \text { mg Magnesium stearate } \\
\text { 109; vaginal capsules; } 7 \text { days; } 6 \\
\text { weeks }\end{array}$ & Clindamycin & $\begin{array}{l}\text { Nugent score } \downarrow \text { by } 5 \text { grades }(P< \\
.001) \\
\downarrow \text { Mean Nugent score }(P<.001) \\
\text { No significant difference in BV } \\
\text { between groups }(P=0.32)\end{array}$ \\
\hline $\begin{array}{l}\text { Larsson } \underline{18} \\
2008 \\
\text { Norway }\end{array}$ & $\begin{array}{l}\text { RCT } \\
100\end{array}$ & $\begin{array}{l}\text { Low } \\
\text { Fair }\end{array}$ & $\begin{array}{l}\text { Combination product }^{d} \\
\text { containing } \\
\text { L gasseri (LBa EB01-DSM 14869) } \\
\text { L rhamnosus (LBp PB01-DSM } \\
\text { 14870) } \\
\text { 108-109; vaginal capsules, } 10 \\
\text { days per month for } 4 \text { months; } 6 \\
\text { months }\end{array}$ & $\begin{array}{l}\text { Metronidazole } \\
\text { (active control } \\
\text { group only) }\end{array}$ & $\begin{array}{l}\uparrow \text { Time to } \mathrm{BV} \text { recurrence }(\mathrm{P}<.027) \\
\text { No significant difference in } \mathrm{BV} \text { cure } \\
\text { rate at one month }(64 \% \text { probiotic vs } \\
78 \% \text { placebo })(P>.05)\end{array}$ \\
\hline $\begin{array}{l}\text { Marcone }{ }^{28} \\
2008 \\
\text { Italy }\end{array}$ & $\begin{array}{l}\text { Prospective } \\
\text { cohort } \\
84 \\
\end{array}$ & $\begin{array}{l}\text { NA } \\
\text { Poor }\end{array}$ & $\begin{array}{l}\text { L rhamnosus } \\
\text { 104; vaginal capsules; } 4 \text { months; } \\
6 \text { months }\end{array}$ & Clindamycin & $\downarrow \mathrm{BV}$ recurrences $(P=.05)$ \\
\hline $\begin{array}{l}\text { Martinez } \underline{\underline{19}} \\
2009 \\
\text { Brazil }\end{array}$ & $\begin{array}{l}\mathrm{RCT} \\
64\end{array}$ & $\begin{array}{l}\text { Unclear } \\
\text { Good }\end{array}$ & $\begin{array}{l}\text { L rhamnosus GR-1 } \\
L \text { reuteri RC-14 } \\
109 \text { each; oral capsules; } 28 \\
\text { days; } 28 \text { days } \\
\end{array}$ & Metronidazole & $\begin{array}{l}\uparrow \mathrm{BV} \text { cure rate }(P=.001) \\
\uparrow \text { Normal Nugent scores }(P=.011)\end{array}$ \\
\hline $\begin{array}{l}\text { Rossi } i^{30} \\
2010 \\
\text { Italy }\end{array}$ & \begin{tabular}{|l} 
Single group \\
40
\end{tabular} & $\begin{array}{l}\text { NA } \\
\text { Poor }\end{array}$ & $\begin{array}{l}\text { L rhamnosus }{ }^{\mathrm{e}} \\
\text { 106; vaginal tablets; } 24 \text { months, } \\
24 \text { months }\end{array}$ & Tinidazole & $\begin{array}{l}\downarrow \mathrm{pH}(P<.001) \\
\text { Change in } \mathrm{pH}(P<.02)\end{array}$ \\
\hline $\begin{array}{l}\text { Ya吕 } \\
2010 \\
\text { China }\end{array}$ & $\begin{array}{l}\text { RCT } \\
120\end{array}$ & $\begin{array}{l}\text { Low } \\
\text { Fair }\end{array}$ & $\begin{array}{l}\text { Combination product } \\
\text { containing } \\
\text { L rhamnosus A-119 } \\
\text { L acidophilus A- } 212\end{array}$ & none & $\begin{array}{l}\downarrow \text { BV recurrences }(P=.001) \\
\downarrow \text { Gardnerella at } 2 \text { months }(P=.02)\end{array}$ \\
\hline
\end{tabular}




\begin{tabular}{|c|c|c|c|c|c|}
\hline & & & $\begin{array}{l}\text { Streptococcus thermophilis A- } \\
336 \\
8 \times 109 \text {; vaginal capsules; } 2 \\
\text { months; } 11 \text { months }\end{array}$ & & \\
\hline $\begin{array}{l}\text { Ling } \underline{29} \\
2013 \\
\text { China }\end{array}$ & $\begin{array}{l}\text { Prospective } \\
\text { cohort } \\
115\end{array}$ & $\begin{array}{l}\text { NA } \\
\text { Fair }\end{array}$ & $\begin{array}{l}\text { L delbrueckjii subsp. lactis } \\
\text { DM8909 } \\
\text { 109; vaginal suppositories; } 10 \\
\text { days; } 1 \text { month }\end{array}$ & $\begin{array}{l}\text { Metronidazole } \\
\text { (active control } \\
\text { group only) }\end{array}$ & $\uparrow \mathrm{BV}$ cure rate at 30 days $(P=.013)$ \\
\hline $\begin{array}{l}\text { Donders } \underline{21} \\
2010 \\
\text { Belgium }\end{array}$ & $\begin{array}{l}\text { RCT } \\
46\end{array}$ & $\begin{array}{l}\text { High } \\
\text { Poor }\end{array}$ & $\begin{array}{l}\text { Combination product } \\
\text { containing } \\
\text { L acidophilus KS400 } \\
\text { plus } 0.03 \text { mg estriol } \\
\text { 107; vaginal suppositories; } 10 \\
\text { days; } 4 \text { months }\end{array}$ & $\begin{array}{l}\text { Metronidazole } \\
\text { (control group only) }\end{array}$ & $\begin{array}{l}\text { Probiotic treatment efficacy } \\
\text { equivalent to metronidazole at } \\
2 \text { weeks, but less effective than } \\
\text { metronidazole at } 1 \text { month. }\end{array}$ \\
\hline $\begin{array}{l}\text { Bradshaw }^{22} \\
2012 \\
\text { Australia }\end{array}$ & $\begin{array}{l}\text { RCT } \\
450\end{array}$ & $\begin{array}{l}\text { Unclear } \\
\text { Fair }\end{array}$ & $\begin{array}{l}\text { Combination product }{ }^{\mathrm{g}} \\
\text { containing } \\
\text { L acidophilus KS } 400 \\
\text { plus } 0.03 \mathrm{mg} \text { estriol } \\
\text { 107; vaginal pessary; } 6 \text { days; } 6 \\
\text { months }\end{array}$ & Metronidazole & $\begin{array}{l}\text { No significant difference in } \mathrm{BV} \\
\text { recurrence between groups }(P=.82)\end{array}$ \\
\hline $\begin{array}{l}\text { Vicariotto }^{\underline{23}} \\
2014 \\
\text { Italy }\end{array}$ & $\begin{array}{l}\mathrm{RCT} \\
35\end{array}$ & $\begin{array}{l}\text { High } \\
\text { Fair }\end{array}$ & $\begin{array}{l}\text { Combination product }^{\mathrm{h}} \\
\text { containing } \\
\text { L fermentum LF15 (DSM 26955) } \\
\text { L planetarium LP01 (LMG P- } \\
21021 \text { ) } \\
340 \mathrm{mg} \text { of arabinogalactan }{ }^{\mathrm{a}} \\
241 \mathrm{mg} \text { of } \\
\text { fructooligosaccharidesa } \\
50 \mathrm{mg} \text { tara gum }{ }^{\mathrm{b}} \\
4 \times 108 \text { each strain; slow- } \\
\text { release vaginal tablet; } 28 \text { days; } 2 \\
\text { months }\end{array}$ & None & $\downarrow$ Nugent scores $(P<.001)$ \\
\hline \multicolumn{6}{|l|}{$\begin{array}{l}\text { Vulvovaginal } \\
\text { Candidiasis }\end{array}$} \\
\hline $\begin{array}{l}\text { Vicariotto }^{31} \\
2012 \\
\text { Italy }\end{array}$ & $\begin{array}{l}\text { Single Group } \\
30\end{array}$ & $\begin{array}{l}\text { NA } \\
\text { Fair }\end{array}$ & $\begin{array}{l}\text { Combination product } \\
\text { containing } \\
\text { L fermentum LF10 }\end{array}$ & None & $\begin{array}{l}\downarrow V V C \text { symptoms at } 1 \text { month }(P< \\
.001) \text { and at } 2 \text { months }(P<.001)\end{array}$ \\
\hline
\end{tabular}




\begin{tabular}{|c|c|c|c|c|c|}
\hline & & & $\begin{array}{l}\text { L acidophilus LA02 } \\
273 \mathrm{mg} \text { of arabinogalactani } \\
332 \mathrm{mg} \text { of } \\
\text { fructooligosaccharides } \\
64 \mathrm{mg} \text { citric acid } \\
56 \mathrm{mg} \text { of sodium bicarbonate } \\
4 \times 108 \text { each strain; slow- } \\
\text { release vaginal tablet; } 28 \text { days; } 2 \\
\text { months }\end{array}$ & & $\begin{array}{l}\downarrow V V C \text { recurrence in } 3 \text { of } 26 \text { at } 2 \\
\text { months }(11.5 \%, P=.083)\end{array}$ \\
\hline $\begin{array}{l}\text { Anukam } \underline{24} \\
2009 \\
\text { Nigeria }\end{array}$ & $\begin{array}{l}\text { RCT } \\
59\end{array}$ & $\begin{array}{l}\text { High } \\
\text { Poor }\end{array}$ & $\begin{array}{l}L \text { rhamnosus GR-1 } \\
L \text { reuteri RC- } 14 \\
5 \times 109 \text { total; oral capsules; } 90 \\
\text { days; } 3 \text { months }\end{array}$ & Fluconazole & $\begin{array}{l}\text { No significant impact of probiotic on } \\
\text { VVC treatment at } 7 \text { days }(P=.149) \\
\downarrow \text { VVC recurrences }(P<.057)\end{array}$ \\
\hline $\begin{array}{l}\text { Murina } 32 \\
2012 \\
\text { Italy }\end{array}$ & $\begin{array}{l}\text { Single group } \\
58\end{array}$ & $\begin{array}{l}\text { NA } \\
\text { Poor }\end{array}$ & $\begin{array}{l}\text { Combination product } \\
\text { containing } \\
\text { L fermentum LF-10 (DSM 19187) } \\
\text { L acidophilus LA02 (DSM 21717) } \\
340 \mathrm{mg} \text { of arabinogalactan } \\
241 \mathrm{mg} \text { of } \\
\text { fructooligosaccharides } \\
50 \mathrm{mg} \text { tara gum } \\
63 \mathrm{mg} \text { citric acid } \\
54 \mathrm{mg} \text { sodium bicarbarbonate } \\
4 \times 10^{8} \text { each strain; slow-release } \\
\text { vaginal tablet; } 28 \text { days; } 7 \\
\text { months }\end{array}$ & $\begin{array}{l}\text { Fluconazole X3 } \\
\text { doses }\end{array}$ & $\begin{array}{l}72 \% \text { experienced no VVC } \\
\text { reoccurrence in } 7 \text { months of follow- } \\
\text { up }\end{array}$ \\
\hline \multicolumn{6}{|l|}{$\begin{array}{l}\text { Urinary Tract } \\
\text { Infection }\end{array}$} \\
\hline $\begin{array}{l}\text { Beerepoot } \underline{25} \\
2012 \\
\text { Netherlands }\end{array}$ & $\begin{array}{l}\text { RCT } \\
252\end{array}$ & $\begin{array}{l}\text { Low } \\
\text { Good }\end{array}$ & $\begin{array}{l}\text { L rhamnosus GR-1 } \\
\text { L reuteri RC-14 } \\
\text { 109; oral capsules; } 6 \text { months; } 15 \\
\text { months }\end{array}$ & $\begin{array}{l}\text { Trimethoprim sulfa- } \\
\text { tamethoxazole }\end{array}$ & $\begin{array}{l}\text { No significant difference in UTI } \\
\text { reoccurrence between probiotics } \\
\text { and controls } \\
\downarrow \text { Mean recurrence per year for } \\
\text { uncomplicated UTI }(P<.001) \\
0.4 \text { UTI per year }(95 \% \mathrm{Cl},-0.4 \text { to } \\
1.5) \text { was outside } 10 \% \text { noninferiority } \\
\text { margin }\end{array}$ \\
\hline
\end{tabular}




\begin{tabular}{|c|c|c|c|c|c|}
\hline & & & & & $\begin{array}{l}\text { Decreased antibiotic resistance in } \\
\text { probiotic group (stats not provided) } \\
\text { No significant difference in AEs (OR, } \\
1.0 ; 95 \% \mathrm{Cl}, 0.6-1.6)\end{array}$ \\
\hline $\begin{array}{l}\text { Stapleton } 26 \\
2011 \\
\text { US }\end{array}$ & $\begin{array}{l}\text { RCT } \\
100\end{array}$ & $\begin{array}{l}\text { Low } \\
\text { Good }\end{array}$ & $\begin{array}{l}\text { Product }^{\text {a }} \text { containing } \\
\text { L. crispatus (CTV-05) } \\
2 \times 10^{9} ; \text { vaginal capsules; } 3 \\
\text { months; } 3 \text { months }\end{array}$ & $\begin{array}{l}\text { Unspecified } \\
\text { "standard } \\
\text { treatment" }\end{array}$ & $\begin{array}{l}\text { Significant difference in } L \text { crispatus } \\
\text { colonization in probiotic group }(P< \\
.004) \\
\text { "High-level colonization with } L \\
\text { crispatus" } \\
\downarrow \text { UTI recurrence }(P<.01)\end{array}$ \\
\hline \multicolumn{6}{|l|}{$\begin{array}{l}\text { Human } \\
\text { Papillomavirus }\end{array}$} \\
\hline $\begin{array}{l}\text { Verhoeven } 27 \\
2013 \\
\text { Belgium }\end{array}$ & $\begin{array}{l}\text { Quasi- } \\
\text { experiment } \\
51\end{array}$ & $\begin{array}{l}\text { NA } \\
\text { Poor }\end{array}$ & $\begin{array}{l}\text { Probiotic beverage }^{k} \text { containing } \\
8 \times 109 \text { L casei Shirota per 2.7- } \\
\text { ounce bottle } \\
\text { Dose not stated; oral drink; } 6 \\
\text { months; } 6 \text { months }\end{array}$ & none & $\begin{array}{l}\text { Twice the clearance of HPV lesions } \\
(P=.05) \\
\text { Probiotic group had increased HPV } \\
\text { viral clearance }(29 \%) \text { vs controls } \\
(19 \%)(P=.41)\end{array}$ \\
\hline
\end{tabular}

Abbreviations: AE, adverse events; BV, bacterial vaginosis; CFU, colony-forming units; HPV, human papillomavirus; $P$, probability; L, Lactobacillus; VVC, vulvovaginal candidiasis; UTI, urinary tract infection.

Notes: aLactin V, Osel, Inc.; Mountain View, CA; bFlorisia, VSL Pharmaceuticals, Inc. Gaithersburg, MD; c Gynophilus, Quamed, Antwep Belgium; dEcoVag, Bifodan, Hunested, Denmark; eNormagin, Baldacci Laboratories; Pisa, Italy; fProbaclac, Nicar Laboratories, Quebec, Canada; gGynoflor, Medinova AG, Zurich, Switzerland; hAcidCand 30, Probiotical, Novara, Italy;iprebiotic fiber; jAlthough the ingredients listed vary slightly between the two publications by Vicariotto,23,31 the use of the same commercially available product was confirmed through personal communication with the primary author; kYakult, Yakult USA, Fountain Valley, CA. 


\section{RESULTS}

In this systematic review, we focused on probiotic interventions as treatment and/or prevention of select urogenital infections in women: BV, candidiasis, UTI, and HPV. In the results section, we report the findings from the studies reviewed in the following order: a) quality assessment; b) study characteristics; c) definitions of urogenital infections; d) participant recruitment, inclusion, exclusion, and study restrictions; e) description of study interventions; f) outcomes by urogenital infection type; and g) adverse events.

\section{Quality Assessment of Randomized Controlled Trials Reviewed}

As shown in Table 1 , we evaluated the 14 RCTs according to risk of bias using the Cochrane assessment criteria 34 and determined that 6 (64\%) were of good quality with a low risk of bias. $\frac{14,16,18,20,25,26}{}$ Five RCTs were rated as having an unclear risk of bias, $, 15,17,19,22$ and the remaining 3 were identified as having a high risk of bias. $\underline{21,23,24}$

The overall quality ratings for the findings of each study reviewed are also presented in Table $\underline{1}$ according to 3 levels within the 3 domains: consistency, directness, and precision. Four studies were determined to have good quality, 9 were fair, and 7 poor, as indicated in the table. Two groups of investigators disclosed relationships with a commercial probiotic financial relationship; others either reported that there was nothing to disclose or did not address the issue. $\underline{14,18}$

\section{Definitions of Urogenital Infections}

The urogenital infections were operationally defined in each study. These diagnoses formed part of the inclusion criteria for participants in the investigations.

Bacterial vaginosis was diagnosed either by Amsel's clinical criteria ( 3 of 4 of the following: homogeneous vaginal discharge, $\mathrm{pH}>4.5$, presence of clue cells, and a positive potassium hydroxide $(\mathrm{KOH})$ amine ("whiff") test) $\underline{36}$ or Nugent's Score $\underline{37}$ (ratings based on a Gram stain smear of vaginal secretions; a score of $\geq 7$ is consistent with BV) or both. Changes in Amsel's criteria and/or Nugent's scores were used as outcome measure(s) to indicate treatment success. Therefore, all of the studies of probiotics for prevention or treatment of BV used appropriate diagnostic criteria.

Vulvovaginal candidiasis was defined as the presence of clinical vaginitis (ie, floccular, odorless vaginal discharge; dyspareunia; dysuria; local irritation) plus evidence of yeast hyphae on microscopic examination, and it can be confirmed by culture using Saboraud agar. $\frac{3,24}{}$ The condition is associated with a normal vaginal $\mathrm{pH}$ of less than or equal to 4.5; therefore, $\mathrm{pH}$ testing is not a useful diagnostic approach for this condition. $\underline{5}$ Lactobacillus colonization is not altered in vulvovaginal candidiasis. $\underline{3}$

Two studies focused on probiotics as an intervention to prevent UTIs. $\frac{25,26}{2}$ Stapleton recruited premenopausal-aged women who had current, uncomplicated, symptomatic cystitis. $\underline{26}$ This was defined as one or more of the following: presence of typical UTI symptoms, 8 or more white blood cells per high-power field on urinalysis, $10^{2}$ or greater CFUs $/ \mathrm{mL}$ of a uropathogen as a single organism. 
Beerepoot recruited asymptomatic women based on their self-reported history of 3 or more symptomatic UTIs in the year prior to the study. $\underline{25}$

Verhoeven studied the efficacy of probiotics to clear HPV infections. $\frac{27}{}$ A finding of LGSIL on the participant's latest Papanicolaou test was used as one of the inclusion criteria for the study. Therefore, in all the studies reviewed, the researchers had used and reported the relevant diagnostic criteria they followed to determine the presence of the infection they were attempting to prevent and/or treat.

\section{Participant Recruitment, Inclusion, Exclusion, and Study Restrictions}

A review of the studies revealed that participants were predominately premenopausal-aged women. In 3 studies, the participants included both pre- and postmenopausal adult women. $\frac{20,30,31}{B e e r e p o o t}$ recruited exclusively postmenopausal participants for a study of probiotics to prevent UTI recurrence. $\underline{25}$ Ling did not describe participant characteristics such as age. $\underline{29}$ In 2 studies, women who had irregular menses were excluded, $\underline{18,26}$ while the timing of menstruation was a consideration in the initiation of the intervention in 6 studies. $\frac{3,15,16,18,19,29}{}$

Several of the investigators added inclusion criteria or restrictions on sexual behavior and/or contraception during the study. In 4 of the studies, women were required to be sexually abstinent during vaginal treatment portions of the study. $\underline{15,16,22,29}$ Hemalatha $\underline{16}$ specifically recruited sexually active participants, while Donders $\underline{21}$ excluded participants who had unprotected intercourse in the prior 24 hours. Restrictions on douching and/or vaginal medications were present for participants in 10 investigations. $\frac{3,15,16,20-23,26,29,31}{\text { In }} 5$ of the studies, participants were excluded if they used certain specified forms of contraception, $\underline{14,16,17,25,28}$ while Stapleton $\underline{26}$ included participants who agreed to use contraception.

\section{Description of Study Interventions}

This section includes our analysis of the study interventions. It refers to information contained in Table 2. The studies were evaluated for the use of medications prescribed for treatment or prevention of the urogynecologic infections before or during the clinical trials. These medications included antimicrobial therapy, antifungals, and estrogen, which are detailed below. 
Table 2. Synthesis of Significant Findings

\begin{tabular}{|c|c|c|c|c|c|}
\hline Urogenital & & Studies Included & $\begin{array}{c}\text { Number } \\
\text { of } \\
\text { Studies }\end{array}$ & & \\
\hline Infection & Goal & in Synthesis & (Na) & Probiotic Intervention Outcome & Strength of Evidence $\underline{b}$ \\
\hline \multirow[t]{6}{*}{$\begin{array}{l}\text { Bacterial } \\
\text { vaginosis }\end{array}$} & Treatment & $\begin{array}{l}\text { Martinez } \underline{19} \text { Ling }^{\underline{29}} \\
\text { Mastromarino } \underline{15}\end{array}$ & $3(231)$ & $\begin{array}{l}\text { Significantly } \uparrow \text { cure: } L \text { rhamnosus and } L \text { reuteri } i^{19} L \\
\text { delbrueckii subsp. lactis } \underline{\underline{29}} \text { A combination product } \underline{15} \\
\text { containing } L \text { brevis } C D 2, L \text { salivarius subsp. salicinius } \\
\text { and } L \text { plantarum (and other active ingredients) }\end{array}$ & $\begin{array}{l}\text { Moderate ; includes } 2 \\
\text { RCTs, one of fair and one } \\
\text { of good quality and one } \\
\text { prospective cohort study } \\
\text { of fair quality }\end{array}$ \\
\hline & Symptoms & Ehrstrom $^{3}$ & $1(95)$ & $\begin{array}{l}\text { Significantly } \downarrow \text { symptoms: } L \text { gasseri, } L \text { fermentum, } L \\
\text { rhamnosus, and } P \text { aciditactici } \underline{3}\end{array}$ & $\begin{array}{l}\text { Low; includes one RCT of } \\
\text { poor quality }\end{array}$ \\
\hline & $\begin{array}{l}\text { Recurrence } \\
\text { rate }\end{array}$ & Marcone,,$\underline{28}$ Donders $\underline{20}$ & $2(204)$ & $\begin{array}{l}\text { Significantly } \downarrow \text { the recurrence rate: } L \text { rhamnosus } s^{28} \text { A } \\
\text { combination product containing } L \text { rhamnosus A-119, } \\
L \text { acidophilus A-212, Streptococcus thermophilis A- } \\
33620\end{array}$ & $\begin{array}{l}\text { Low, includes one RCT of } \\
\text { poor quality and a } \\
\text { prospective cohort study } \\
\text { of fair quality }\end{array}$ \\
\hline & $\begin{array}{l}\text { Recurrence } \\
\text { time }\end{array}$ & - $\quad$ Larsson $\underline{18}$ & $1(100)$ & $\begin{array}{l}\text { Significantly } \uparrow \text { time to recurrence: A combination } \\
\text { product } \frac{18}{18} \text { containing } L \text { gasseri (LBa EB01-DSM 14869) } \\
\text { L rhamnosus (LBp PB01-DSM 14870) }\end{array}$ & $\begin{array}{l}\text { Low; includes one RCT of } \\
\text { fair quality }\end{array}$ \\
\hline & $\mathrm{pH}$ level & - $\operatorname{Rossi}^{30}$ & $1(40)$ & $\begin{array}{l}\text { Significantly } \downarrow \text { vaginal } \mathrm{pH} \text { compared to } \mathrm{pH} \text { tablet: } \mathrm{L} \\
\text { rhamnosus } \stackrel{30}{=}\end{array}$ & $\begin{array}{l}\text { Low; includes one single } \\
\text { group study of poor } \\
\text { quality }\end{array}$ \\
\hline & $\begin{array}{l}\text { Nugent } \\
\text { scores }\end{array}$ & $\begin{array}{l}\text { Martine } \underline{19}^{\underline{\underline{9}}} \text { Vicariotto } \underline{\underline{23}} \\
\text { Mastromarino } \underline{15} \\
\text { Petricevic } \underline{\underline{17}}\end{array}$ & $4(323)$ & $\begin{array}{l}\text { Signficantly } \downarrow \text { Nugent scores: } L \text { rhamnosus and } L \\
\text { reuteri } \underline{19} L \text { fermentum and } L \text { planetarum } m^{23} \mathrm{~A} \\
\text { combination product } \underline{15} \text { containing } L \text { brevis } \mathrm{CD} 2, L \\
\text { salivarius subsp. salicinius and } L \text { plantarum (and } \\
\text { other active ingredients) A combination product } \frac{17}{\underline{1}} \\
\text { containing } L \text { casei rhamnosus (Lcr35) (and another } \\
\text { active ingredient) }\end{array}$ & $\begin{array}{l}\text { Moderate; includes } 3 \\
\text { RCTs, } 2 \text { with fair and one } \\
\text { with good quality }\end{array}$ \\
\hline \multirow[t]{2}{*}{$\begin{array}{l}\text { Vulvovaginal } \\
\text { candidiasis }\end{array}$} & Symptoms & $\begin{array}{l}\text { - } \quad \text { Vicariotto }{ }^{31} \\
\text { - } \\
\text { Ehrstrom }{ }^{3}\end{array}$ & $2(140)$ & $\begin{array}{l}\text { Significantly } \downarrow \text { symptoms: A combination product } \\
\text { containing } L \text { fermentum } \mathrm{LF} 10, L \text { acidophilus } \mathrm{LA02} \text {, and } \\
\text { other ingredients }{ }^{31} \text { L gasseri, } L \text { fermentum, } L \\
\text { rhamnosus, and Pediococcus acidilactici }{ }^{\underline{3}}\end{array}$ & $\begin{array}{l}\text { Low, includes one RCT of } \\
\text { poor quality and one } \\
\text { single group study of fair } \\
\text { quality }\end{array}$ \\
\hline & Prevention & - Anukum $^{24}$ & 1(59) & $\begin{array}{l}\text { Significantly } \downarrow \text { recurrences: A combination product } \\
\text { containing } \underline{24} \text { L rhamnosus A-119, } L \text { acidophilus A-212, } \\
\text { Streptococcus thermophilis A-336 }\end{array}$ & $\begin{array}{l}\text { Low; includes one RCT of } \\
\text { poor quality }\end{array}$ \\
\hline
\end{tabular}




\begin{tabular}{|l|l|l|l|l|l|}
\hline $\begin{array}{l}\text { Urinary tract } \\
\text { infection }\end{array}$ & Prevention & $\begin{array}{l}\text { Beerepoot } \underline{\underline{25}} \\
\text { Stapleton } \underline{\underline{26}}\end{array}$ & 2(352) & $\begin{array}{l}\text { Significantly } \downarrow \text { mean annual recurrence: } L \\
\text { rhamnosus and } L \text { reuteri } \underline{25} \text { Significant } \downarrow \text { in } \\
\text { recurrence: High-level colonization with } L \text { crispatus } \underline{26}\end{array}$ & $\begin{array}{l}\text { Moderate; includes } 2 \\
\text { RCT of good quality }\end{array}$ \\
\hline HPV & $\begin{array}{l}\text { Lesion } \\
\text { resolution }\end{array}$ & $\bullet$ Verhoeven $\underline{27}$ & $1(51)$ & $\begin{array}{l}\text { Twice the clearance of LGSIL: Ingestion of a milk } \\
\text { product containing L. casei Shirota } \underline{\underline{27}}\end{array}$ & $\begin{array}{l}\text { Low; includes one quasi- } \\
\text { experiment of poor } \\
\text { quality }\end{array}$ \\
\hline
\end{tabular}

Abbreviations: L, Lactobacillus; HPV, human papillomavirus; UTI, urinary tract infection; LGSIL, low-grade squamous intraepithelial lesions.

${ }^{a}$ Sample sizes from individual studies are included in more than one probiotic intervention outcome, if applicable.

${ }^{b}$ Adapted from strength of evidence used from the Agency for Healthcare Research and Quality Methods Guide. $\frac{35}{}$ Definitions from Likis et al: $\underline{33}$

"High: High confidence that the evidence reflects the true effect. Further research is unlikely to change estimates. Moderate: Moderate confidence that the evidence reflects the true effect. Further research may change our confidence in the estimate of effect and may change the estimate.

Low: Low confidence that the evidence reflects the true effect. Further research is likely to change confidence in the estimate of effect and is also likely to change the estimate.

Insufficient: Evidence is either unavailable or does not permit a conclusion" (p. ES-9).

\section{Antimicrobial Therapy}

Antimicrobial therapy varied among studies but was used in most. Among the 14 investigations of BV, 5 used no conventional antibiotic

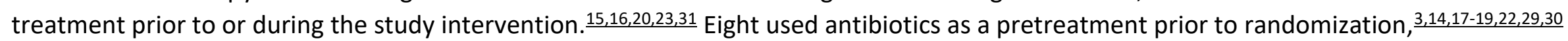
while Donders $\underline{21}$ treated concurrently with an antibiotic and the probiotic study intervention. Ling conducted a cohort study in which participants with BV were either treated using metronidazole gel or the vaginal probiotic intervention. $\underline{\underline{29}}$

Antimicrobial interventions varied between the 2 probiotic investigations on UTIs. Stapleton recruited participants with an acute UTI and randomized them to a placebo or probiotic intervention after all received standard antibiotic treatment. $\underline{26}$ In a noninferiority trial, Beerepoot compared antimicrobial therapy to a probiotic intervention to suppress UTI recurrences. $\underline{25}$

\section{Antifungal Therapy}

Anukam enrolled women with a history of recurrent candidiasis. All participants were randomized following conventional treatment to either a placebo or probiotic. $\underline{.4}$ Murina studied the efficacy of a commercially available probiotic product after all participants received 3 doses of fluconazole (Table $\underline{2}) \cdot \underline{32}$

\section{Estriol Therapy}

Two of the probiotic interventions for BV included $0.03 \mathrm{mg}$ of vaginal estriol with the probiotic. $\underline{21,22}$ This was a part of one of the commercially available probiotic combination products noted by Donders only. $\underline{21}$ Rationale for the estriol component of the intervention was not provided. These researchers did not report participants' use of any other estrogen-containing products. 


\section{Selection and Type of Probiotic Interventions}

The studies were reviewed to evaluate the type of probiotic interventions used. All of the study interventions included a single or multiple strain combination of Lactobacillus species (see Table 2 ). Although multiple species were used, $L$ rhamnosus and $L$ acidophilus were the most frequently used probiotics. Ya added Streptococcus thermophilis to 2 Lactobacillus strains. $\underline{20}$ Ehrstrom used a combination that

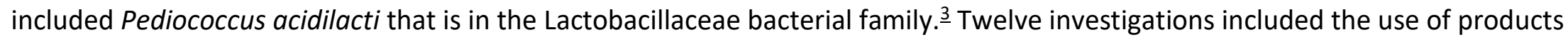
commercially available in their study settings. The ingredients of these commercially available products are detailed in Table $\underline{2}$. Although 3

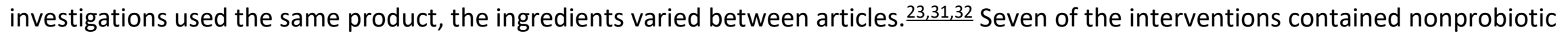
active ingredients.

Several investigators provided a rationale for choice of probiotic(s) species and/or strains that was specific for action against the urogenital infection studied. Hermmerling conducted preparatory in vitro testing and determined that $L$ crispatus CTV-05 was effective against BV. $\underline{14}$ Stapleton chose the same probiotic for its proven efficacy against BV. $\underline{26}$ Petrievic used $L$ casei rhamnosus Lcr 35 for its 20 -year record of safety and its proven ability to inhibit Gardnerella vaginalis. 17 Murina indicated that the choice of product was based on in vitro testing that demonstrated efficacy on the vaginal microflora without fostering candidial propagation. $\underline{32}$ Bradshaw stated that $L$ acidophilus KS400 was chosen because of prior research that demonstrated efficacy against BV. $\underline{22}$ Hemalatha and Ya selected probiotic strains with general evidence of efficacy against urogenital infections. $\frac{16,20}{2}$ Ling referred to in vitro testing for efficacy of $L$ delbrueckii subspecies lactis DM8909 against BV but did not provide a citation. $\underline{29}$

Ehrstrom stated that Pediococcus acidilactici LN23 was chosen for BV and candidiasis because it has a shorter generation time compared to lactobacilli. It was hypothesized that this would lead to a rapid decrease in vaginal pH because it would act as starter culture for other vaginal probiotic bacteria. Vicariotto selected the strain $L$ plantarum LF5 based on in vitro testing that demonstrated its inhibition of Gardnerella vaginalis. $\underline{23}$ Beerepoot used a combination of $L$ rhamnosus GR-1 and $L$ reuteri RC-14, because of evidence of efficacy with restoration of vaginal flora and to reduce colonization with pathogens. $\underline{25}$ Slow release, effervescent vaginal tablets containing citric acid and sodium bicarbonate were used by Vicariotto and Murina to administer the probiotic. $\frac{31,32}{2}$ Their rationale was that the commercial product would rapidly (40-60 minutes) create an anaerobic vaginal environment to foster probiotic growth while creating a biofilm to prevent pathogen adherence. $\frac{31,32}{}$

\section{Dosing}

The probiotic dosing was clearly stated in 13 of the 20 studies reviewed (65\%) as presented in Table $\underline{2}$. Dosages ranged from $10^{4}$ to $10^{10}$ CFUs. Most investigators reported daily doses between $10^{7}$ and $10^{9} \mathrm{CFUs}$ for the probiotic interventions. Verhoeven did not provide the probiotic intervention dose used. $\underline{27}$ Beerepoot did not clarify whether the dose provided was for each individual probiotic or if they were 
reporting a total of the CFUs in the combination product. $\stackrel{25}{5}$ Several other investigators stated a range of dosing for the probiotics they had studied but never gave an exact dose. $\frac{3,15,16,18}{2}$

\section{Route}

Sixteen of the studies used the vaginal route for the probiotic interventions. Of these, 5 used vaginal capsules exclusively, $\frac{3,17,18,20,26}{8}$ employed vaginal tablets, $\underline{15,16,21,23,28,30-32}$ one a vaginal applicator, $\underline{14}$ one vaginal suppositories, $\underline{\underline{29}}$ and one a vaginal pessary. $\underline{\underline{22}}$ Three investigations used oral capsules for the probiotic intervention. $\underline{19,24,25}$ Verhoeven delivered the intervention in a commercially available probiotic beverage (Table $\underline{1}) \cdot \underline{27}$

\section{Length of Treatment}

The duration of the probiotic interventions ranged from 5 days to 12 months, as shown in Table $\underline{2}$. The studies aimed at prevention had somewhat longer probiotic interventions. Rationale for the duration of treatments was not provided in the studies reviewed.

\section{Outcomes}

The evaluation of outcomes was complicated by the variations in probiotic interventions, duration of treatment, and the goals of treatment, prevention, or both. Significant probiotic related findings of the studies are summarized by urogynecologic infection in Table $\underline{2}$. The efficacy of the probiotic intervention was evaluated in each study using the diagnostic criteria for the specific infection(s) as described in the study design (eg, a decreased Nugent score for BV).

\section{Bacterial Vaginosis}

An examination of the 14 investigations of probiotics against BV revealed that 8 studies were focused on treatment of BV, 3,14-16,19,21-23 while 6 focused on prevention. 17,18,20,28-30 However, some of the treatment-related trials reported outcomes on recurrences. Therefore, the lines separating treatment and prevention were indistinct.

All of the investigations of probiotics against BV included premenopausal-aged participants. As shown in Table 2 , Martinez, Ling, and Mastromarino all reported a significant increase in BV cure in the probiotic group compared to controls. $\frac{15,19,29}{20 t}$ all of the investigations use BV cure as their main study outcome. For example, Hemmerling $\underline{14}$ measured vaginal colonization with the probiotic bacteria. Rossi $\underline{30}$ evaluated $\mathrm{pH}$ by using a $\mathrm{pH}$ tablet as control and found that the probiotic intervention resulted in both a significant reduction in vaginal $\mathrm{pH}$ compared to controls at measurement points, as well as a significant change in $\mathrm{pH}$ from baseline. In 4 studies, a decrease in the Nugent score was the primary or secondary study outcome. $\frac{15,17,19,23}{}$ Both Marcone $\frac{28}{}$ and $\mathrm{Ya}^{20}$ found that the probiotic interventions reduced BV recurrence while Larsson $\frac{18}{}$ found that the probiotic intervention significantly reduced the time to BV recurrence. Hemalatha demonstrated that the probiotic intervention significantly reduced 2 proinflammatory cytokines. $\underline{16}$ 


\section{Vulvovaginal Candidiasis}

Among the 3 investigations of probiotics to treat and/or prevent candidiasis, findings were variable, as shown in Table $\underline{2}$. None of the studies demonstrated that the probiotic interventions were effective in the treatment of acute candidiasis. However, both Anukam $\underline{24}$ and Vicariotto $\underline{31}$ found a significant reduction in recurrences in women who received the probiotic intervention. Ehrstom found that probiotic bacteria were still detectable at 6 months in $9 \%$ of the intervention group. $\underline{3}$

\section{Mixed Urogenital Infections}

Ehrstrom also explored the impact of a probiotic intervention for women who had either BV or candidiasis or both. $\frac{3}{-}$ The probiotic intervention significantly increased colonization with lactic acid bacteria and led to significant reductions in symptoms.

\section{Urinary Tract Infections}

The 2 double-blind placebo controlled RCTs of probiotics to prevent UTI recurrences were examined for commonalities and differences. $\frac{25,26}{2}$ Stapleton (2011) demonstrated that the probiotic intervention significantly increased colonization with $L$ crispatus and this led to a reduction in UTI recurrence..$^{26}$ Beerepoot (2013) found that the probiotic intervention did not meet the noninferiority margin, but among women with uncomplicated UTI, the average number of UTI recurrences was significantly less in the probiotic group compared to controls. This association was not found for women with complicated UTIs. Additionally, antibiotic resistance increased in the control group but not in the probiotic group participants. $\frac{25}{}$

\section{Human Papillomavirus}

In a quasi-experimental pilot of $L$ casei Shirota, a probiotic drink was used in participants who had an LGSIL on their Papanicolaou test. $\underline{27}$ The intervention resulted in significant clearance of HPV lesions, as shown in Table $\underline{2}$. While viral clearance was reduced, it was not statistically significant.

\section{Length of Follow-up}

Follow-up also varied significantly among investigations from one to 11 months. Investigators used various approaches for more long-term follow-up periods. For example, Bradshaw $\underline{22}$ used self-collected swabs returned by mail to the researchers, while Yaํㅡ evaluated long-term outcomes of BV recurrence through the use of phone calls to collect self-reports.

\section{Adverse Events}

Thirteen of the studies reviewed indicated that adverse events monitoring was done, 3,14-16,18-22,24-26,31 but 6 of these provided either no reports or the information was unclear. $\frac{15,16,19,20,26,32}{}$ Two investigators relied on participant self-reports,, 22 while Hemmerling conducted detailed data collection. $\underline{14}$ Overall, there were no significant differences in the incidence of adverse events among probiotic and control 
group participants in any of the RCTs that included this analysis. The use of effervescent vaginal tablets led to at least one dropout for vaginal burning, which could be considered a minor adverse event. $\underline{32}$ None of the studies reported serious adverse events.

\section{DISCUSSION}

Although clinical practice recommendations were limited by the strength of evidence, probiotic interventions were effective in treatment and prevention of urogenital infections as alternatives or cotreatments. The synthesized findings presented in Table $\underline{2}$ provide insights that may be useful to women's health care providers who wish to apply probiotics for treatment and/or prevention of various urogynecologic infections.

\section{Study Bias and Quality}

Tracking of primary and even secondary outcomes compared to study aims was confounded by a lack of precision and clarity in a number of the studies reviewed. Some of the investigators who conducted RCTs calculated a priori sample sizes by determining the effect size for their primary outcome variable. Those who did not report such calculations tended to have small sample sizes that precluded statistical analysis beyond simple frequencies and percentages. More well-controlled studies with sample sizes based on the effect size of the primary outcome variable are needed to further develop the body of literature.

\section{Limitations}

It was challenging to analyze and synthesize study findings because of the heterogeneity in design, methods, and study outcomes, including those reporting on the same infection. Only 6 of the RCTs reviewed had low risk of bias as assessed by Cochrane Collaboration Risk of Bias of Randomized Controlled Trials Assessment. $\underline{34}$ Our quality ratings allowed us to review all 20 studies for more than risk of bias. However, the small number of high-quality studies limited determinations about the effectiveness of the interventions in the studies reviewed. More well-controlled trials that specify a clear purpose and a primary outcome variable that is measurable are needed. It is important for researchers to describe the probiotic intervention fully and to provide participants with clear and realistic instructions to avoid undue burden.

\section{Probiotic Interventions}

Several investigators provided a specific rationale for the choice of probiotic intervention, some based on in vitro testing of the probiotic bacteria against particular organisms. The rationale for dosing and/or the treatment duration were not provided in studies. There were significant variations among the probiotic interventions, such as strain, species, dosage, frequency, routes of administration, and treatment duration. Even when a common species, such as Lactobacillus acidophilus, was used, the variety of different strains made comparisons difficult. The properties of one strain may not necessarily be attributed to another. $\underline{10}$ Even when 2 authors reported using the same 
commercially available probiotic product, there were variations in ingredient details. The use of commercially available products as standardized probiotic interventions may allow for the study of the same species, strains, and additional ingredients. These uniform products hold promise, but their use may be limited by their accessibility in various countries. The variability among studies concerning all aspects of the probiotic interventions used limited the generalizability and utility of the study outcomes. More research on well-identified strains tested with larger samples will build a stronger body of knowledge and allow for meta-analyses to inform both research and clinical practice.

\section{Probiotic Intervention Outcomes}

While outcomes varied among studies on probiotics used to treat or prevent the same urogenital infections, a pattern of significant findings emerged. Table $\underline{2}$ contains a synthesis of significant findings. Authors of a Cochrane Review of probiotics for the treatment of BV, $\underline{38}$ that included investigations outside of the search years for this systematic review, found that probiotic interventions provided beneficial effects when given in combination with metronidazole (odds ratio [OR], 0.09; 95\% confidence interval [Cl], 0.03-0.26) or when combined with estriol (OR, 0.02; 95\% Cl, 0.00-0.47).

Although there is no standard, both Reid $\underline{39}$ and Andreu $\underline{40}$ recommended doses between $10^{9}$ to $10^{11} \mathrm{CFUs}$ by any route for probiotic interventions. In most of the studies, the probiotic intervention was delivered via the vaginal route. Although both oral and vaginal dosing have been found to be effective in the management of urogenital infections, the ideal route remains unclear. $\underline{41}$

Outcomes that addressed the mechanisms of action of probiotics were reported in several studies. The bacteria in the probiotic intervention colonized the vagina within 2 to 10 days of administration. $\frac{3,14,26}{2}$ This colonization may sustain beyond the duration of the active probiotic intervention. $\frac{3,24}{}$ The finding that the intervention decreased 2 proinflammatory cytokines addresses the beneficial immunologic actions of probiotics. $\frac{16}{1}$ In one study, the probiotic intervention acidified the vaginal mucosa more than $\mathrm{pH}$ tablets. $\frac{30}{}$ These outcomes may serve to guide future research as well as clinical practice.

Several of the findings were not statistically significant, yet are clinically relevant. For example, Donders found that a combination product containing $10^{7} \mathrm{CFU} L$ acidophilus (KS400) plus $0.03 \mathrm{mg}$ estriol was equivalent to metronidazole for the short-term treatment of BV. $\underline{21}$ The consumption of a probiotic milk product containing $8 \times 10^{9} \mathrm{CFU} L$ casei Shirota per 2.7-ounce bottle was associated with an increase in HPV viral clearance. $\underline{27}$ In general, HPV infections can spontaneously resolve within 2 years in greater than $79 \%$ of individuals, with variations depending on HPV type, the presence of coinfections with multiple types, and behavioral factors such as smoking. $\frac{42}{}$ The pilot study finding that probiotic intervention resulted in significant HPV lesion clearance deserves further attention and study in an RCT. Although spontaneous LGSIL resolution is possible, the authors of a prospective study identified this occurred in fewer than $20 \%$ of participants. $\frac{43}{}$ 
Lesion clearance is dependent on the same factors that impact viral clearance. $\frac{42,43}{}$ More well-designed and controlled research studies on the outcomes of probiotic interventions against HPV infections are needed to verify these findings.

None of the investigators reported any serious adverse events attributable to the probiotic intervention. Adverse events did not differ significantly between groups in any of the RCTs. Probiotic interventions are generally regarded as safe in healthy populations because probiotics are not systemically absorbed. $\underline{44}$ Active and complete monitoring for adverse events in future investigations will help strengthen the body of knowledge regarding probiotic safety.

\section{Antibiotic Treatment}

The use and timing of antibiotics in relationship to the probiotic intervention varied considerably among studies. The intricacies of participant instructions for antibiotic versus probiotic timing were only specified in one study. $\underline{25}$ Antibiotics can significantly disrupt the normal vaginal flora. $\underline{45}$ Probiotics must withstand digestion to be effective on mucosal surfaces, such as the vagina. $\underline{11}$ If antibiotics and probiotics were administered simultaneously, the antibiotic could destroy the probiotic bacteria in the gastrointestinal tract. .6 Separation of antibiotics and probiotics doses by 2 to 4 hours is recommended for this reason. $\underline{46}$ It is plausible that more investigations would have had significant findings if antibiotic and probiotic interventions were carefully timed and clearly reported. The prevalent use of antibiotics in treatment groups may have limited the findings attributable to the probiotic interventions.

Standard antibiotic treatments of infections varied among studies and did not always meet the CDC guidelines for treatment adequacy. For example, in China, Ling $\underline{29}$ used metronidazole once daily for 7 days to treat BV as an active control; however, the CDC recommends twicedaily treatment. $\underline{2}$

\section{Intimate Behaviors of Participants}

Stark contrasts were found among studies on participant recruitment, inclusion, exclusion, and study restrictions. Exclusions of sexually active or sexually abstinent women, or women who did or did not use contraception, provide examples of these variations. Although the vagina is an adaptive ecosystem, unprotected sexual intercourse and menstrual variations lead to fluctuations in the physiology. Douching and vaginal medications can disrupt vaginal homeostasis. $\underline{38}$ As presented in the findings, several investigators conducted data collection on these variables throughout their investigations. Close control of study variables increases the internal validity in RCTs. However, this analysis demonstrated the complexity of enrolling female participants and monitoring their intimate behaviors. More research that balances the need for control with the realities of women's lives will help inform clinical practice. 


\section{CONCLUSION}

The quality of the studies in this systematic review varied. Although we cannot make substantial clinical practice recommendations based on the strength of evidence, probiotics may be an alternative or co-treatment of urogenital infections. Women's health care practitioners can use the available evidence to discuss treatment options with women who wish to avoid antibiotics, or who want to use probiotics to address their health care needs.

Probiotic interventions were shown to have some efficacy in the treatment and prevention of urogynecologic infections. More wellcontrolled investigations are needed to ensure sufficient sample size for statistical analyses, with consistent inclusion of rationale for researchers' choices of probiotics, dosing, procedures, adjunctive antibiotic therapy, and outcome measures whenever possible. In addition, clarity about the use of randomization, blinding, and other strategies to increase quality and reduce risk of bias is needed in future work.

Given the imperative to reduce antibiotic use to avoid the increasing development of resistance, patients and clinicians need alternative approaches to treat and prevent common urogenital infections. Probiotic manufacturers might be considered as viable sources of support for clinical research, as long as these relationships have well-documented boundaries and are clearly documented. Although probiotic availability has increased, science has to keep pace with generating the necessary knowledge through research to support evidence-based clinical practice.

\section{CONFLICT OF INTEREST}

The authors have no conflicts of interest to disclose.

Continuing education units (CEUs) are available for this article as a part of a continuing education theme issue. To obtain CEUs online, please visit www.jmwhce.org. A CEU form that can be mailed or faxed is available in the print edition of this issue.

\section{Biographies}

- Lisa Hanson, CNM, PhD, FACNM, is a Professor and Director of the Midwifery Program at the Marquette University, College of Nursing, Milwaukee WI. She is a staff nurse-midwife at the Aurora Midwifery and Wellness Center.

- Leona VandeVusse, CNM, PhD, FACNM, is an Associate Professor at the Marquette University, College of Nursing, Milwaukee, Wisconsin.

- Martha Jermé, RN, MSN, MLIS, is Health Sciences Librarian at Marquette University, Raynor Memorial Libraries.

- Cybéle Lara Abad, MD, is a Clinical Associate Professor at the University if the Philippines, Philippine General Hospital, Manila. 
- Nasia Safdar, MD, PhD, is an Associate Professor at the University of Wisconsin School of Medicine and Public Health, Department of Medicine and Infectious Disease, Madison, Wisconsin, and Chief of Staff for Research at the William S. Middleton Memorial Veterans Hospital.

\section{REFERENCES}

1 Friedman CR, Whitney CG. It's time for a change in practice: reducing antibiotic use can alter antibiotic resistance. J Infect Dis. 2008;197:1082-1083.

2 Centers for Disease Control \& Prevention [CDC]. 2015 Sexually Transmitted Diseases Treatment Guidelines-Bacterial Vaginosis. Atlanta, GA: Centers for Disease Control \& Prevention; 2015, June 4. http://www.cdc.gov/std/tg2015/bv.htm Accessed August 15, 2015.

3 Ehrstrom S, Daroczy K, Rylander E, et al. Lactic acid bacteria colonization and clinical outcome after probiotic supplementation in conventionally treated bacterial vaginosis and vulvovaginal candidiasis. Microb Infect. 2010;12:691-699.

4 Bradshaw CS, Morton AN, Hocking J, et al. High recurrence rates of bacterial vaginosis over the course of 12 months after oral metronidazole therapy and factors associated with recurrence. J Infect Dis. 2006;193:1478-1489.

5 Centers for Disease Control \& Prevention [CDC]. 2015 Sexually Transmitted Diseases Treatment Guidelines -Vulvovaginal Candidiasis. Atlanta, GA: Centers for Disease Control \& Prevention; 2015, June 4. http://www.cdc.gov/std/tg2015/candidiasis.htm. Accessed August 30, 2015.

6 Hooton TM, Gupta K. Acute uncomplicated cystitis and pyelonephritis in women. In: Calderwood SB, ed. UpToDate. Waltham, MA: Wolters Kluwer; 2015. http://www.uptodate.com/contents/acute-uncomplicated-cystitis-and-pyelonephritis-inwomen?source=search_result\&search=Hooton+TM\%2C+Gupta+K.+Acute+uncomplicated+cystitis+and+pyelonephritis+in+women.\& selectedTitle=1\%7E150. Accessed August 31, 2015.

7 Castle PE. The life cycle, natural history, and immunology of human papillomaviruses. In: Hirsch MS, ed. UpToDate. Waltham, MA: Wolters Kluwer; 2015. http://www.uptodate.com/contents/the-life-cycle-natural-history-and-immunology-of-humanpapillomaviruses?source=search_result\&search=Castle+PE.++The+life+cycle\%2C+natural+history\%2C+and+immunology+of+human+ papillomaviruses. Accessed August 31, 2015.

8 Bolton M, Van Der Straten A, Cohen CR. Probiotics: potential to prevent HIV and sexually transmitted infections in women. Sex Transm Infect. 2008;35(3):214-225.

9 Donders GG. Microscopy of the bacterial flora on fresh vaginal smears. Infect Dis Obstet Gynecol. 1999;7:177-179.

10 Reid G, Dols J, Miller W. Targeting the vaginal microbiota with probiotics as a means to counteract infections. Curr Opin Clin Nutr Metab Care. 2009;12(6):583-587.

11FAO/WHO Working Group Report on Drafting Guidelines for the Evaluation of Probiotics in Food. Guidelines for the Evaluation of Probiotics in Food. London, Ontario, Canada: Author; April-May 2002. http://www.fda.gov/ohrms/dockets/dockets/95s0316/95s0316-rpt0282-tab-03-ref-19-joint-faowho-vol219.pdf. Accessed July 4, 2015. 
12 Abad CL, Safdar N. The role of Lactobacillus probiotics in the treatment or prevention of urogenital infections: A systematic review. J Chemother. 2009;21(3):243-252.

13 Moher D, Liberati A, Tetzlaff J, Altman DG. The PRISMA Group (2009) Preferred Reporting Items for Systematic Reviews and MetaAnalyses: the PRISMA Statement. PLoS Med. 2009;6(7):e1000097.

14 Hemmerling A, Harrison W, Schroeder A, et al. Phase 2a study assessing colonization efficiency, safety, and acceptability of Lactobacillus crispatus CTV-05 in women with bacterial vaginosis. Sex Transm Dis. 2010;37(12):745-750.

15 Mastromarino P, Macchia S, Meggiorini L, et al. Effectiveness of lactobacillus-containing vaginal tablets in the treatment of symptomatic bacterial vaginosis. Clin Microbiol Infec. 2009;15(1):67-74.

16 Hemalatha R, Mastromarino P, Ramalaxmi BA, Balakrishna NV, Sesikeran B. Effectiveness of vaginal tablets containing lactobacilli versus pH tablets on vaginal health and inflammatory cytokines: A randomized, double-blind study. Eur J Clin Microbiol Infect Dis. 2012;31:3097-3105.

17 Petricevic L, Witt A. The role of Lactobacillus casei rhamnosus Lcr35 in restoring the normal vaginal flora after antibiotic treatment of bacterial vaginosis. Br J Obstet Gynaecol. 2008;115(11):1369-1374.

18 Larsson P-G, Stray-Pedersen B, Ryttig KR, Larsen S. Human lactobacilli as supplementation of clindamycin to patients with bacterial vaginosis reduce the recurrence rate: a 6-month, double-blind, randomized, placebo-controlled study. BMC Womens Health. 2008;8(3):[8].

19 Martinez RCR, Franceschini SA, Patta MC, et al. Improved cure of bacterial vaginosis with single dose of tinidazole ( $2 \mathrm{~g}$ ), Lactobacillus rhamnosus GR-1, and Lactobacillus reuteri RC-14: a randomized, double-blind, placebo-controlled trial. Can J Microbiol. 2009;55:133-138.

20 Ya W, Reifer C, Miller LE. Efficacy of vaginal probiotic capsules for recurrent bacterial vaginosis: a double-blind, randomized, placebocontrolled study. Am J Obstet Gynecol. 2010;203(2):120e1-120e6.

21 Donders GGG, Bulck B, Van de Walle P, et al. Effect of lyophilized lactobacilli and 0.03 mg estriol (Gynoflor ${ }^{\circledR}$ ) on vaginitis and vaginosis with disrupted vaginal microflora: a multicenter, randomized, single-blind, active-controlled pilot study. Gynecol Obstet Invest. 2010;70(4), 264-272.

22 Bradshaw CS, Pirotta M, de Guingand D, et al. Efficacy of oral metronidazole with vaginal clindamycin or vaginal probiotic for bacterial vaginosis: randomised placebo-controlled double-blind trial. PLoS ONE. 2012;7(4):e34540.

23 Vicariotto F, Mogna L, Del Piano M. Effectiveness of the two microorganisms Lactobacillus fermentum LF15 and Lactobacillus plantarum LP01, formulated in slow-release vaginal tablets, in women affected by bacterial vaginosis: a pilot study. J Clin Gastroenterol. 2014;48:S106-S112.

24 Anukam KC, Duru MU, Eze CC, et al. Oral use of probiotics as an adjunctive therapy to fluconazole in the treatment of yeast vaginitis: a study of Nigerian women in an outdoor clinic. Microb Ecol Health D. 2009;21(2):72-77.

25 Beerepoot MAJ, Ter Riet G, Nys S, et al. Lactobacilli versus antibiotics to prevent urinary tract infections: a randomized, double-blind, noninferiority trial in postmenopausal women. Arch Intern Med. 2012;172(9):704-712. 
26 Stapleton AE, Au-Young M, Hooton TM, et al. Randomized, placebo-controlled Phase 2 trial of a Lactobacillus crispatus probiotic given intravaginally for the prevention of recurrent urinary tract infection. CID. 2011;52(10): 1212-1217.

27 Verhoeven V, Renard N, Makar A, et al. Probiotics enhance the clearance of human papillomavirus-related cervical lesions: a prospective controlled pilot study. Eur J Cancer Prev. 2013;22(1):46-51.

28 Marcone V, Calzolari E, Bertini M. Effectiveness of vaginal administration of Lactobacillus rhamnosus following conventional metronidazole therapy: how to lower the rate of bacterial vaginosis recurrences. New Microbiol. 2008;31(3):429-433.

29 Ling Z, Liu X, Chen W, et al. The restoration of the vaginal microbiota after treatment for bacterial vaginosis with metronidazole or probiotics. Microb Ecol. 2013;65(3):773-780.

30 Rossi A, Rossi T, Bertini M, Caccia G. The use of Lactobacillus rhamnosus in the therapy of bacterial vaginosis: evaluation of clinical efficacy in a population of 40 women treated for 24 months. Arch Gynecol Obstet. 2010;281:1065-1069.

31 Vicariotto F, Del Piano M, Mogna L, Mogna G. Effectiveness of the association of 2 probiotic strains formulated in a slow release vaginal product, in women affected by vulvovaginal candidiasis: a pilot study. J Clin Gastroenterol. 2012;46:S73-S80.

32 Murina F, Graziottin A, Vicariotto F, De Seta F. Can Lactobacillus fermentum LF10 and Lactobacillus acidophilus LA02 in a slow-release vaginal product be useful for prevention of recurrent vulvovaginal candidiasis? A clinical study. J Clin Gastroenterol. 2014;48(Suppl.1):S102-S105.

33 Likis FE, Andrews JC, Collins MR, et al. Nitrous oxide for the management of labor pain. Comparative Effectiveness Review No. 67. AHRQ Publication No. 12-EHC071-EF. Rockville MD: Agency for Healthcare Research and Quality; 2012. Website www.effectivehealthcare.ahrq.gov/reports/final.cfm. Accessed December 15, 2015.

34 Higgins JPT, Altman DG, Gotzsche PC, et al. The Cochrane Collaboration's tool for assessing risk of bias in randomized trials. BMJ. 2011;343:d5928.

35 Agency for Healthcare Research and Quality (AHRQ). Methods Guide for Effectiveness and Comparative Effectiveness Reviews. Rockville, MD: AHRQ; 2008-2011. http://www.ncbi.nlm.nih.gov/books/NBK47095/. Accessed September 1, 2015.

36 Amsel R, Totten PA, Spiegel CA, Chen KC, Eschenbach D, Holmes KK. Nonspecific vaginitis: diagnostic criteria and microbial and epidemiologic associations. Am J Med. 1983;74:14-22.

37 Nugent RP, Krohn MA, Hillier SL. Reliability of diagnosing bacterial vaginosis is improved by a standardized method of gram stain interpretation. J Clin Microbiol. 1991;29:297-301.

38 Senok AC, Verstraelen H, TemmermanM, Botta GA. Probiotics for the treatment of bacterial vaginosis. Cochrane Database Syst Rev. 2009;4:CD006289.

39 Reid G, Charbonneau D, Erb J, et al. Oral use of Lactobacillus rhamnosus GR-1 and L. fermentum RC-14 significantly alters vaginal flora: randomized, placebo-controlled trial in 64 healthy women. Fems Immunol Med Mic. 2003;35(2):131-134.

40 Andreu A. Lactobacillus as a probiotic for preventing urogenital infections. Rev Med Microbiol. 2004;15:1-6.

41 Huang $\mathrm{H}$, Song L, Zhao W. Effects of probiotics for the treatment of bacterial vaginosis in adult women: a meta-analysis of randomized clinical trials. Arch Gynecol Obstet. 2014;289(6):1225-1234. 
42 Jaisamrarn U, Castellsagué X, Garland SM, et al. Natural history of progression of HPV infection to cervical lesion or clearance: analysis of the control arm of the large, randomised PATRICIA Study. PLoS One. 2013;8(11):e79260.

43 Woo YL, van den Hende M, Sterling JC, Colema N. A prospective study on the natural course of low-grade squamous intraepithelial lesions and the presence of HPV16 E2-, E6- and E7-specific T-cell responses. Int J Cancer. 2010;126:133-141.

44 Elias J, Bozzo P, Einarson A. Are probiotics safe for use during pregnancy and lactation? Can Fam Phys. 2011;57:299-301.

45 Sullivan A, Edlund C, Nord CE. Effect of antimicrobial agents on the ecological balance of human microflora. Lancet Infect Dis. 2001;1:101-114.

46 Boyanova L, Mitov I. Coadministration of probiotics with antibiotics: why, when and for how long? Expert Rev Anti Infect Ther. 2012;10(4):407-409. http://www.ncbi.nlm.nih.gov/pubmed/22512748. Accessed September 1, 2015. 